\title{
Programmable Transmission Systems using Coherent Detection enabling Multi-Tb/s Interfaces for IT-communications Convergence in Optical Networks
}

\author{
Josep M. Fabrega ${ }^{a}$, Michela Svaluto Moreolo ${ }^{\mathrm{a}}$, Laia Nadal ${ }^{\mathrm{a}}$, Ricardo Martínez ${ }^{\mathrm{a}}$, \\ Christian Neumeyr ${ }^{\mathrm{b}}$, Alberto Gatto ${ }^{\mathrm{c}}$, Paola Parolaric ${ }^{\mathrm{c}}$, Mariangela Rapisarda ${ }^{\mathrm{c}}$, and \\ Pierpaolo Boffic
}

${ }^{a}$ Centre Tecnològic de Telecomunicacions de Catalunya (CTTC/CERCA), Castelldefels, Spain ${ }^{\mathrm{b}}$ Vertilas GmbH, Garching, Germany

'Politecnico di Milano, Dip. Elettronica, Informazione e Bioingegneria, Milano, Italy

\begin{abstract}
The information technologies (ITs)-communications infrastructure convergence is key to future optical networks for achieving an automated orchestration of IT, optical and cloud resources. To cope with this challenge, transmission systems need to be agile, programmable, and capable of transmitting large amounts of data, while covering data center and metropolitan networks. This work addresses transmission solutions using coherent detection combined with either direct or external modulation, with special focus on flexible, high capacity and cost/energy-efficient systems. The proposed technological solutions are evaluated in terms of throughput, and programmability and interaction with the control plane.
\end{abstract}

Keywords: Sliceable bandwidth/bitrate variable transceiver (BVT), digital signal processing (DSP), vertical cavity surface emitting laser (VCSEL), multicarrier modulation

\section{INTRODUCTION}

Future network services are conceived around the joint use of different heterogeneous resources (including transport, fixed and mobile), while combining networking and cloud functions. For example, different core functionalities can be hosted in data centers located at different local metro nodes and close to the end users. This entails IT-communications infrastructure convergence, e.g. inspired by the habits of cloud providers, inside their data centers, which will be expanding to all devices, users, and machines worldwide. Therefore, a unified optical connectivity as a service for everything is needed, enabling the automated orchestration of IT, optical and cloud resources.

In order to tackle such a huge challenge, dynamic data plane solutions are needed. In fact, transmission systems need to be agile, programmable, and capable of transmitting large amounts of data quickly, while enabling the suitable allocation and configuration of bandwidth resources accordingly. Furthermore, these transmission systems are expected to be cost/power-effective and cope with different distance ranges, covering actual data center and metropolitan networks.

In this work, we introduce the main use case in a generic network scenario, highlighting its needs. Furthermore, we also present the intensity modulation transmission solutions using coherent detection combined with either direct or external modulation, with special focus on flexible, high capacity and cost-effective systems. ${ }^{1}$ The performance and optimization of the proposed technologies is evaluated by experiments. Additionally, we discuss programmability and flexibility trade-offs when interacting with the control plane. 


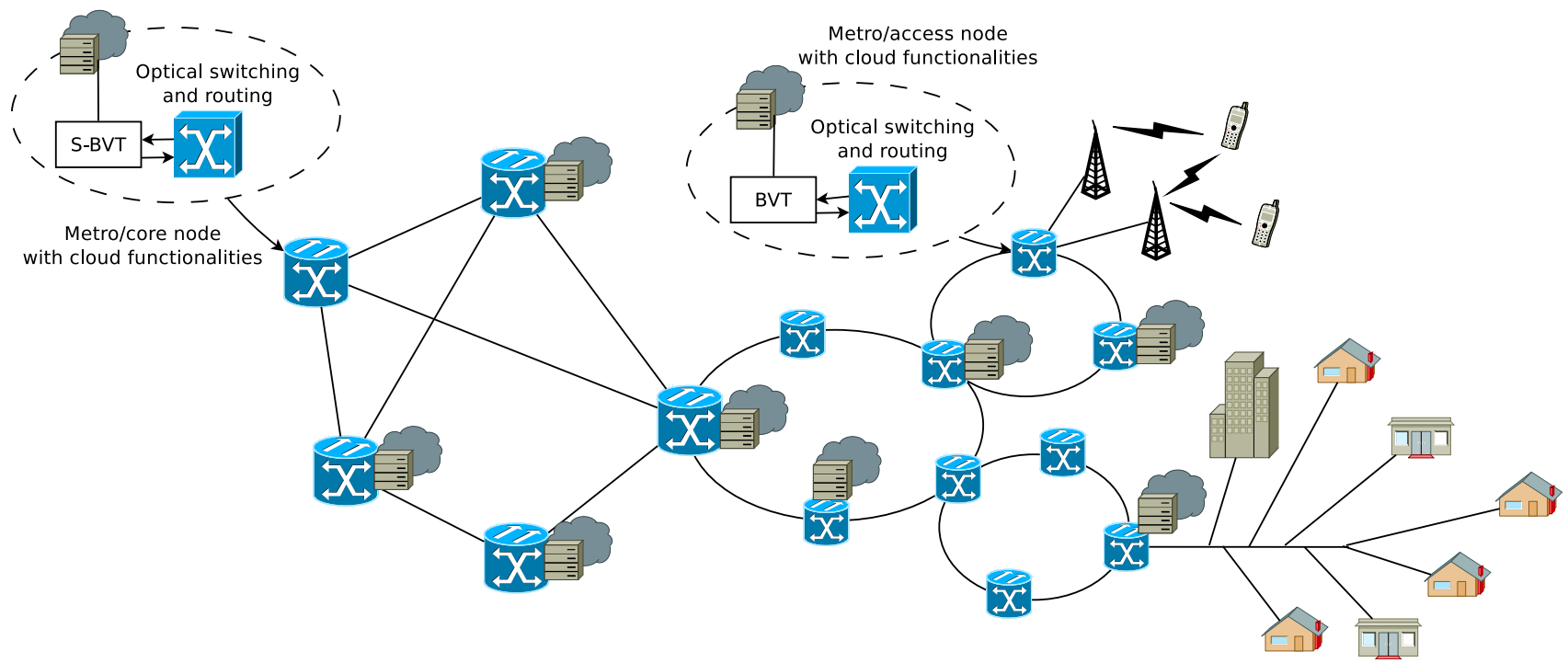

Figure 1. Generic network scenario for the proposed IT-telecom convergence.

\section{NETWORK SCENARIO AND MAIN CONCEPT}

The envisioned network scenario is depicted in Fig. 1, which can be regarded as a straightforward step beyond contemporary fiber optic networks. There, the main change is not on the architecture itself, which is still expected to feature different levels of aggregation, but the inclusion of different computing (IT) resources at each optical node in order to support different applications and services. In fact, network function virtualisation and disaggregated computing are becoming the basis for the functions and services that networks offer. ${ }^{2}$ So, the computing resources hosted in different nodes can be expected to work as a distributed data centers, with enhanced computing capacity and stringent intra/inter-connection needs. For example, they can be used for video caching (in TV on demand), intensive computing, hosting virtual network functions, etc.

Since the IT resources are expected to be present at almost each optical node, a way to save capital expenditure (CapEx), and even operating expenses (OpEx), can be to merge IT and telecommunications network elements into a single converged infrastructure. This network would act seamlessly as telco infrastructure, giving a connectivity to both, the network users and the IT resources. This unified optical connectivity would be possible by reusing the existing fiber deployments of the operators and simply adapting the different nodes to this paradigm.

As shown in Fig. 1, the nodes include different network elements: a pool of IT computing resources, a set of transceivers, BVTs, and an optical switching unit for traffic aggregation, disaggregation and routing. So in such a converged infrastructure we expect that the same network elements (and even physical devices) can be used for delivering traffic in the datacenter of each node, between different datacenters (located at different nodes) and/or providing connectivity to the different end users. In order to cope with that, it is expected to also have increased flexibility and programmability of the network at different levels. In fact, we could expect different flows with different capacity and different service level agreement to coexist in the very same infrastructure for achieving the desired automated orchestration of IT, optical and cloud resources.

This leads to different traffic patterns and data plane requirements that should be met by the same network elements and resources. Regarding the former, network disaggregation and slicing are key, since they enable to operate the network at both perspectives using the same infrastructure, provided that there is enough flexibility in the data plane. ${ }^{3}$ In the data plane, the focus should be put on maximizing performance, versatility and, most importantly, cost-efficiency. As a result, photonic technologies need to support ultra-dynamic reconfigurations for lasers, cross-connects, optical amplifiers, for on-chip or stand-alone components. So, network systems, subsystems

Further author information: (Send correspondence to J. M. Fabrega)

J. M. Fabrega: E-mail: jmfabrega@cttc.es 
and devices are expected to be programmable and controllable, eventually leveraging network analytics, in order to create a truly flexible and smart infrastructure.

Among all the network elements, the most important ones are the transceivers. These transmission solutions should tackle with increased robustness against common impairments (e.g. chromatic dispersion, polarization mode dispersion, fiber nonlinear effects,...) in transmission distances covering up to several hundreds of $\mathrm{km}$. Furthermore, a truly flexible, scalable, high-capacity and cost-efficient solution should be pursued in order to support different aggregated data traffic volumes while eventually operating at heterogeneous granularities corresponding to the different aggregation levels of the network. Among the possible alternatives, the so-called BVTs are the ideal solution. In fact, the BVTs can be remotely configured by the control plane, for an optimal management of the network resources. ${ }^{4}$ In addition to the bitrate variability that can cope with a dynamic traffic variation (e.g. daily traffic variation), the BVTs also feature other benefits also there are other benefits such as the capability to attain a specific capacity demand for a given connection. ${ }^{4}$ Additionally, the BVTs can be stacked in order to act as a single virtual transceiver, the sliceable bandwidth/bitrate variable transceiver (SBVT), so that it can be shared to concurrently serve more than one connection. Therefore, multiple paths can be assigned to a given S-BVT, in order to keep it highly utilized and reduce the total number and cost of transmission solutions. ${ }^{5}$ The parameters to be configured at each BVT include wavelength, spectral occupancy and modulation format/power per flow. So, the proposed BVTs deliver data flows with variable spectral occupancy and rate, according to the network and path conditions.

Among all the options for implementing the BVTs, those based on orthogonal frequency-division multiplexing (OFDM) are the most attractive for cost-effectively coping with the flexibility requirements of elastic optical networks. ${ }^{5}$ In fact, OFDM provides advanced spectrum manipulation capabilities, including arbitrary sub-carrier suppression and bit/power loading. Thanks to these features, OFDM transceivers can be ad hoc configured for achieving a certain reach and/or coping with a targeted data rate as expected. However, the main question here is to reduce the cost as much as possible while being able to keep a high performance in the broad range of connectivity cases (from intra-datacenter up to metropolitan/regional segments). Therefore, a simple intensity modulation combined with coherent detection is proposed. On the one side, intensity modulation enables the use of compact and low cost modulation schemes, including direct modulation laser (DML). On the other side, coherent detection improves the transmission performance, even for intensity modulation, and all the main opti$\mathrm{cal} /$ electronic devices can be integrated in a photonic integrated circuit. For example, in the PASSION project it is envisioned to use a VCSEL-based modular S-BVT architecture combined with coherent reception, able to support up to $2-\mathrm{Tb} / \mathrm{s}$ per module. ${ }^{6}$

\section{TRANSCEIVER}

The scheme of the basic BVT unit is shown in Fig. 2. It is based on OFDM featuring intensity modulation and coherent reception.

At the transmitter, incoming data are first processed at the OFDM DSP stage in order to generate a digital OFDM waveform. So, data are parallelized and mapped into the corresponding M-level quadrature amplitude modulation (QAM) constellation. Adaptive bit/power loading can be implemented for either optimizing the performance at a given capacity, or optimizing capacity at a given performance. For example, the rate adaptive version of the Levin-Campello algorithm ${ }^{7}$ can be used in order to test the maximum capacity that the BVT can offer. Then, training symbol (TS) are included for performance monitoring and extracting the channel response at the receiver side. The resulting symbols feed an inverse fast Fourier transform (IFFT). Afterwards, a cyclic prefix $(\mathrm{CP})$ is added and the obtained OFDM symbols are then serialized and upsampled. The digital OFDM signal is clipped and upconverted to an intermediate frequency of half of its bandwidth by mixing with a digital oscillator. The resulting signal is pre-emphasized and converted to the analog domain by a digital to analog converter (DAC). This signal drives the transmission optoelectronic front-end, which can be based either on external modulation, e.g. a Mach-Zehnder modulator (MZM) feeded by a tunable lightwave source (TLS), or a DML, e.g. a simple cost-effective VCSEL. In order to improve the transmission performance, an optical bandpass filter (OBPF) can be used for obtaining an optical single side band (SSB) signal.

In case of using a DML, the laser bandwidth might be rather limited in comparison to external modulation. So, when employing a DML the edge of the intensity response of the laser is expected to be smoothly decreasing 

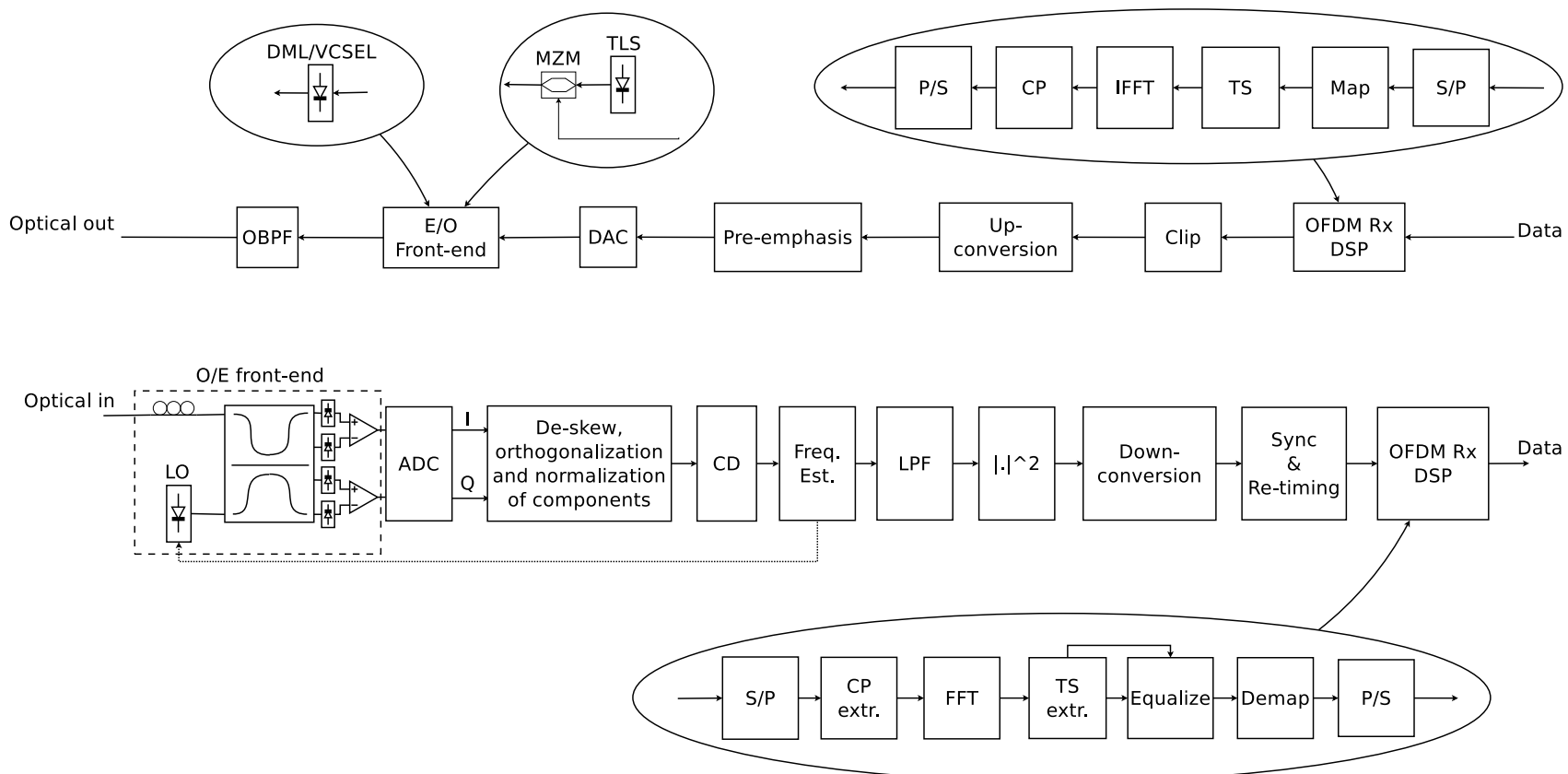

Figure 2. Transceiver scheme.

when increasing the frequency. Therefore, adaptive modulation is very much indicated in this case, since it enables to locally use constellations featuring low bits per symbol. Also, pre-emphasis filtering equalizes the intensity response of the laser by giving and increased gain at high frequencies with respect to low frequencies.

At the receiver, an optoelectronic front-end based on coherent reception is proposed. For the sake of simplicity, in this work we only consider a single-polarization scheme based on 90-degree phase diversity. Therefore, the received signal is appropriately polarized and interfered in a 90-degree hybrid by a local laser, which acts as a local oscillator (LO). At the output of the hybrid, two balanced detection pairs drive a two-channel analog to digital converter (ADC). The LO is set at a frequency so that the electrical signals are centred in baseband. Therefore, the ADC bandwidth can be minimized also thanks to the fact of detecting an optical SSB signal. The digitized in-phase and quadrature (I/Q) signals are first de-skewed, orthogonalized and normalized in order to optimally combine them, obtaining a proper complex representation of the received signal. Afterwards, chromatic dispersion is compensated, by employing a suitable digital filter, and the frequency difference between LO and received signal is estimated. This value is then driving the LO in order to compensate the laser frequency drift and obtain an accurate baseband representation of the received signal. A digital low pass filter (LPF) is applied and the square modulus function is applied in order to obtain a representation of the power of the received signal. Then the OFDM signal is downconverted to baseband, and a sync and retiming (downsampling) procedure is applied to obtain a suitable baseband OFDM signal. Finally, the baseband OFDM signal is demodulated following the reversal of the OFDM modulation above described.

De-skew, orthogonalization and normalization of the I/Q components is key for the performance of any coherent receiver. Of course, when employing an integrated receiver front-end is more advantageous, since delays in the different paths of the balanced detectors can be appropriately designed and manufactured. However, when using a receiver build up with discrete components this module turns up to be of high importance for compensating the differences between I/Q components in order to obtain a proper complex representation of the received signal.

One of the key modules of the receiver is the LPF. This filter is specifically designed to avoid unwanted mixing products between LO and received signals, while rejecting as much noise as possible. Therefore, its response is expected to feature sharp edges and a rather limited bandwidth. So, the LO needs to be able to provide an accurate frequency difference between LO and received signals. 

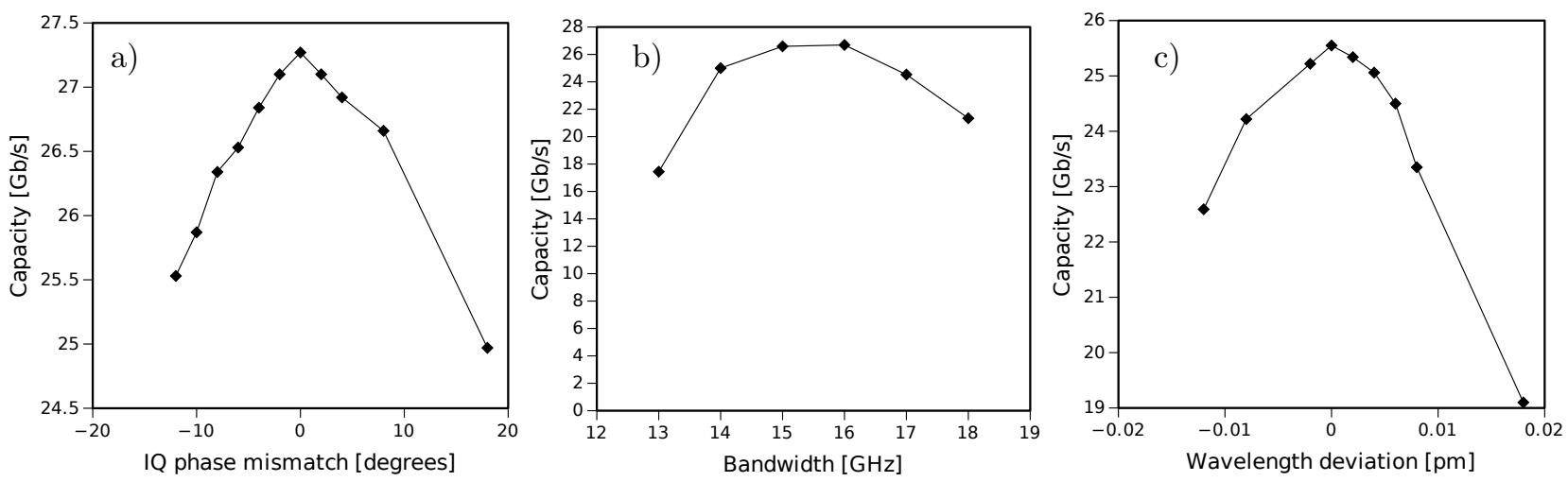

Figure 3. Experimental performance optimization of the receiver. System capacity as function of the I/Q phase mismatch (a), of the LPF bandwidth (b), and of the wavelength difference between the carrier of the received signal and the LO.

\section{PERFORMANCE OPTIMIZATION}

In this section we report an initial performance optimization of the key receiver parameters in terms of capacity in a back-to-back scenario with $30 \mathrm{~dB}$ optical signal to noise ratio (OSNR). The capacity is maximized using the Levin-Campello rate adaptive loading algorithm, using the suitable margins in order to obtain a a bit error ratio (BER) between $10^{-3}$ and $4.6 \cdot 10^{-3}$. There, the OFDM modulation signal is of $18 \mathrm{GHz}$ bandwidth, while the laser is a VCSEL of around $10 \mathrm{GHz}$ bandwidth. ${ }^{8}$

First, we test the robustness against phase mismatch between I and Q components. Results are shown in Fig. 3a. There we can see that maximum capacity is decreased only by a $5 \%$ for a phase mismatch below 8 degrees. Therefore, the tolerance is quite reasonable, as could expected in a transmission based on intensity modulation where no information is encoded in the phase of the optical signal.

Next, we test the optimal LPF bandwidth. These results are depicted in Fig. 3b. In this case, the maximum capacity is achieved when approaching $15.2 \mathrm{GHz}$. This is slightly higher than the bandwidth of the VCSEL employed, meaning that the VCSEL features a smoothly decreasing frequency response after $10 \mathrm{GHz}$. Therefore, we can take advantage of that when using adaptive OFDM modulation. ${ }^{8}$

Finally, we optimize the LO wavelength in terms of capacity. This is shown in Fig. 3c, where we can observe a $10 \%$ capacity decrease for a frequency detuning of only $12 \mathrm{pm}$ (around $1.5 \mathrm{GHz}$ ). Since VCSELs are typically a bit unstable in terms of frequency drift, this means that the LO should feature a high wavelength accuracy for attaining a good performance.

After this optimization, the transceiver is tested in a laboratory testbed and compared to a simple direct detection, showing that at OSNR values below $25 \mathrm{~dB}$, the BVT capacity is increased thanks to the coherent reception. ${ }^{8}$ Furthermore, in a $160-\mathrm{km}$ link featuring $26.4 \mathrm{~dB}$ OSNR; the developed coherent receiver supports $22.6 \mathrm{~Gb} / \mathrm{s}$, while the maximum capacity achieved with direct detection is lower than $10 \mathrm{~Gb} / \mathrm{s}$.

\section{PROGRAMMABILITY}

Despite the inherent flexibility of the solution, and some basic programmable features like capacity, there are several other parameters and functions that could be adopted and/or configured by the network control plane. In terms of the parameters, it would be much easy and simple for the transceiver if the control plane could specify the transmission distance and the type of fiber(s) employed in order to perform a suitable dispersion compensation at the receiver side. This could be key, as the control plane is expected to have a complete knowledge of the network, not only in terms of actual performance but also in terms of the available optical resources.

Furthermore, several data plane functions could be adopted by the network controller in order to maximize the performance according to the traffic needs. Since fiber-optic networks are known to be quite stable (e.g. in the range of minutes), the network controller could perform several non-intensive functions typically associated to 
the data plane, but including the knowledge of the status of the network and the different optical channels. These functions could include the bit/power loading algorithm, the calculus of pre-emphasis filter at the transmitter, and/or the low-pass/equalization filter parameters at the receiver.

In order to include these functions in the network controller, a precise performance monitoring should be deployed. In fact, this can be performed at the transceivers themselves. For example, the frequency response of the channel to the OFDM waveform can be acquired by means of the training symbols introduced at the transmitter. However, this gives a channel response with limited accuracy. A much precise channel response can be acquired by transmitting a long OFDM waveform featuring uniform bit/power loading. Alternatively, since an intensity modulation is pursued, the channel response can be directly acquired by a high resolution spectrum analysis. $^{9}$

\section{CONCLUSIONS}

In this work we have introduced the IT-communications infrastructure convergence as key paradigm to future optical networks for achieving an automated orchestration of IT, optical and cloud resources. To cope with this challenge, transmission systems need to be agile, programmable, and capable of transmitting large amounts of data, while covering data center and metropolitan networks. Therefore, we have presented BVT-based transmission solutions employing OFDM intensity modulation and coherent detection. The proposed technological solutions have been discussed in terms of performance optimization and programmability.

\section{ACKNOWLEDGMENTS}

The work reported in this paper is funded in part by the Spanish AURORAS project (RTI2018-099178-B-I00) and the European Union's Horizon 2020 research and innovation programme under grant agreement PASSION no. 780326 .

\section{REFERENCES}

[1] Svaluto Moreolo, M., Nadal, L., Fabrega, J. M., Vilchez, F. J., Casellas, R., Muñoz, R., Neumeyr, C., Gatto, A., Parolari, P., and Boffi, P., "Modular SDN-enabled S-BVT Adopting Widely Tunable MEMS VCSEL for Flexible/Elastic Optical Metro Networks," in [2018 Optical Fiber Communications Conference and Exposition (OFC)], 1-3 (March 2018).

[2] Photonics21 Public Private Partnership, "Photonics21 Multiannual Strategic Roadmap 2021-2027," (2019).

[3] Riccardi, E., Gunning, P., de Dios, O. G., Quagliotti, M., López, V., and Lord, A., "An Operator view on the Introduction of White Boxes into Optical Networks," Journal of Lightwave Technology 36, 3062-3072 (Aug 2018).

[4] Sambo, N., Castoldi, P., D'Errico, A., Riccardi, E., Pagano, A., Moreolo, M. S., Fabrega, J. M., Rafique, D., Napoli, A., Frigerio, S., Salas, E. H., Zervas, G., Nolle, M., Fischer, J. K., Lord, A., and Giménez, J. P. F. P., "Next generation sliceable bandwidth variable transponders," IEEE Communications Magazine 53, 163-171 (Feb. 2015).

[5] Svaluto Moreolo, M., Fabrega, J. M., Nadal, L., Vílchez, F. J., Mayoral, A., Vilalta, R., noz, R. M., Casellas, R., Martínez, R., Nishihara, M., Tanaka, T., Takahara, T., Rasmussen, J. C., Kottke, C., Schlosser, M., Freund, R., Meng, F., Yan, S., Zervas, G., Simeonidou, D., Yoshida, Y., and Kitayama, K.-I., "SDN-enabled sliceable BVT based on multicarrier technology for multiflow rate/distance and grid adaptation," Journal of Lightwave Technology 34, 1516-1522 (Mar. 2016).

[6] Gatto, A., Parolari, P., Rapisarda, M., Neumeyr, C., Bhat, S., Delrosso, G., Moreolo, M. S., Fabrega, J. M., Nadal, L., and Boffi, P., "Disruptive photonic technologies for the future sustainable high-capacity metro network," in [2019 21st International Conference on Transparent Optical Networks (ICTON)], 1-4 (July 2019).

[7] Nadal, L., Svaluto Moreolo, M., Fàbrega, J. M., Dochhan, A., Grießer, H., Eiselt, M., and Elbers, J.-P., "DMT modulation with adaptive loading for high bit rate transmission over directly detected optical channels," $J$. Lightwave Technol. 32, 3541-3551 (Nov 2014). 
[8] Svaluto Moreolo, M., Nadal, L., Martínez, R., Casellas, R., Vilchez, F. J., Muñoz, R., Vilalta, R., Gatto, A., Parolari, P., Boffi, P., Neumeyr, C., Larrabeiti, D., Otero, G., and Fernández-Palacios, J. P., "Experimental Assessment of a Programmable VCSEL-based Photonic System Architecture over a Multi-hop Path with 19-Core MCF for Future Agile Tb/s Metro Networks," in [2020 Optical Fiber Communications Conference and Exposition (OFC)], 1-3 (March 2020).

[9] Fabrega, J. M., Svaluto Moreolo, M., Nadal, L., Vilchez, F. J., Villafranca, A., and Sevillano, P., "Experimental study of adaptive loading in im/dd ofdm using in-band optical sub-carrier snr monitoring," in [Optical Fiber Communication Conference], Optical Fiber Communication Conference, Th2A.12, Optical Society of America (2016). 